\title{
Unquestionable Wound Pain: Call for A Care-Plan Reform
}

\author{
Joanne Lusher $^{1 *}$, Alexandra Murrell ${ }^{1}$, Betty Changa ${ }^{1}$, Christopher Mafuva ${ }^{1}$, Eddy Awire ${ }^{1}$ and David Chapman Jones ${ }^{2}$ \\ ${ }^{1}$ University of the West of Scotland, London Campus, UK
}

${ }^{2}$ Great Ormond Street Hospital NHS Foundation Trust and Institute of Child Health University College London. UK

*Corresponding author: Joanne Lusher, University of the West of Scotland, London Campus, UK

\section{Opinion}

Pain is a subjective experience and one that the medical healthcare profession will never fully be able to understand because the pain belongs to the patient. Ultimately, pain is whatever the patient experiences it to be, yet the traditional medical model approach is one that views the patient's pain as something to be managed by the 'professional' [1,2]; who have been reported to often mismanage it [3]. Pain is in fact a personal encounter and one that is perceived differently both within and between individuals from one time to the next. This point must not only be acknowledged, but digested and acted upon, by healthcare providers working within the field of pain medicine. This paper concedes that pain is a biopsychological event that can only be fully managed by adopting a patient-centered and holistic approach to care. This proposition extends its focus to draw particular attention to those working in the field of wound care, due to the unique pain-related idiosyncrasies apparent among patients with acute and chronic wounds (from any origin: war/accident trauma; postoperative; foot/leg ulcers; burns/scars) that make them worthy of special attention in this matter.

In most recent years, wounds have been estimated to cost health services up to $\$ 96$ billion globally [4] and this this figure is only set to rise due to self-compromising lifestyle and behavioral factors that lead to wound-promoting illnesses (for example obesity and diabetes) [5]. Pain has traditionally and consistently been dismissed as an overlooked aspect in wound care [6], yet pain has a detrimental and often devastating impact on wound healing [7] that negatively compromises an already weakened immune system [8]. Stress further perpetuates this deleterious cycle of events that tamper with effective wound healing, yet pain management does not take precedence in standard 'wound care' regimes. To illustrate, on reviewing the literature on various pain management guides, protocols and strategies specific to wound care published over the past twenty years (2000-2020), it became evident that, as with other types of pain, wound pain continues to be managed principally by pharmacological methods [9-12]. That is, guidance being offered from various commanding sources, from the World Health Organisation (WHO) to specialist wound and pain advocates [10-12] typically recommends a prescription care package of antiinflammatory, non-steroidal medication (ibuprofen), which might be escalated to an oral pain killer (codeine) if the pain is greater and a stronger opiate analgesic (morphine) is offered when the pain becomes more severe. What this approach does is treat the symptom so is therefore less than optimum, because pain is more than just a symptom. Pain intensity, severity and duration are not only governed by physiological causes, but a psychological response. It has been reported that pain causes stress and stress negatively impacts healing [13]. Furthermore, pain perception (and therefore wound healing) is further compounded by anxiety; depression; and sleep deprivation as further by-products of stress [7].

Not only does this interacting biopsychosocial spiral of events impact on wound healing, but when it comes to pain from a wound, this topic becomes ever more complex due to the unique ways in which pain is manifested among patients with wounds. What makes wound pain distinct from pain caused by other illnesses (for instance cancer or arthritis), is that wound pain is consistently and blatantly visible to the sufferer. What this does is provides a constant reminder and red flag to notify the individual that there is something wrong. In fact, research has shown that wound pain is highest when dressings are removed [13], as opposed to being hidden by a dressing; and even when bandaged, the dressing remains a constant reminder of the fact that there is cause for concern. This form of biofeedback acts by conditioning a patient to a sense of heightened stress and unease that becomes amplified by an anticipatory response. This has been witnessed during such 
times when the wound becomes re-exposed, such as during dressing changes [13] and independently of pain sensation resulting from tissue disruption. A conditioned response therefore develops to prompt the physical system to experience pain. This anticipatory reaction explains pain perception as a bi-directional response. Not only can the physical body trigger warnings to the psychological self that there is a problem, but the mind can alert the body to feel pain. This mind-body dualism is magnified in the case of wound pain due to the high visibility of the source of the pain in question and this is precisely why pain regulation should be a focal point in any wound care plan due to its idiosyncratic qualities.

In order to bring pain medicine and treatment practice up to speed with contemporary integrated pain models [13], we need to consider holistic care packages that strategically intervene on both the physical and psychological levels of concern [6]. For example, empowering patients to self-manage their illness [1,14] both practically (e.g. wound dressing; patient-controlled analgesia) and mentally (e.g. cognitive reframing; stress management). Moreover, 'treating' the psychology should form an integrated part of any standard care package for wound patients [15], whereby continuous dialogue is used between patient and healthcare provider to monitor and reflect on aspects of the regimen. Patient pain diaries and monitoring of thoughts, feelings and behaviors can be achieved through use of self-support cognitive behavioral training for wound pain patients that facilitate restructuring negative coping strategies such as catastrophizing and fear self-statements [1].

Overall, it is aspired that this debate will provoke the attention of professionals working with patients with painful conditions to reconsider, firstly, that there is more to treating pain than a numbing of the symptom. As we appreciate, pain is an incredibly complex experience that cannot be managed reliably with medication alone. Secondly, wound pain is distinctive in that pain perception can be magnified by the unconcealed nature (or root cause) of the pain, which is unlike most other painful conditions. Finally, we wish readers to ponder over these germane issues right now, because despite witnessing scatterings within the literature of debates relating to the issues addressed here, it can be argued confidently, that all concerted efforts to get this message across to inform treatment plans thus far have failed, as we still do not see these viewpoints translated in to practice. We concede by reiterating that pain is subjectively owned by the patient and is therefore an un-questionable experience so can never be fully understood by a person outside of that experience; and call for a care-plan reform that guarantees a fully-integrated, biopsychosocial tailored package that responds to the voice of each patient.

\section{References}

1. Lusher J, Murray E (2018) Contested pain: Managing the invisible symptom. International Journal of Global Health 1-2.

2. Chapman Jones D, Lusher J (2019) Possible wider implications and clinical value of commercially sponsored evaluations: Discussion on recent methodology. Journal of Wound Care 28(8): 1-8.

3. Brown A (2014) Strategies to reduce or eliminate wound pain. Nursing Times 110(15): 12-15.

4. Nussbaum S, Carter MJ, Fife CE, DaVanzo J, Haught R et al. (2018) An economic evaluation of the impact, cost and medicare policy implications of chronic, non-healing wounds. Value Health 21(1): 27-32.

5. Lusher J, Murray E, Chapman Jones D (2017) Changing the way we think about wounds: A challenge for 21st Century medical practice. International Wound Journal 15(2): 311-312.

6. Bechert K, Abraham S (2009) Pain management and wound care. Journal of the American College of Certified Wound Specialists 1(2): 65-71.

7. Lusher J, Chapman Jones D (2019) Biological clocks: Is it time for a closer watch on skin healing? Global Journal of Anaesthesia and Pain Medicine 1(4): 73-75.

8. Kiecolt Glaser J, Marucha PT, Malarkey WB, Mercado AM, Glaser R (1995) Slowing of wound healing by psychological stress. Lancet 346(8984): 1194-1196.

9. World Health Organisation (2020) Web statement on Pain management guidance.

10. Wright K and Shirey J (2003) Addressing the pain: A pain management protocol for wound care. Wound Management and Prevention 49: 5.

11. Suzuki K, Zoe Birnbaum BS, Ryan Lockhart (2016) Principles in pain management for wound care patients. Podiatry Today 29(8): 58-61.

12. Sussman C, Bates-Jensen B (2011) Wound care: A collaborative practice manual for health professionals. Baltimore: Lippincott, Williams and Wilkins.

13. Woo K (2012) Exploring the effects of pain and stress on wound healing. Advances in Skin and Wound Care 25(1) 38-44.

14. Lusher J (2020) Who's wound is it anyway? International Wound Journal, in press.

15. Lusher J, Djatmika C (2020) Living with a Caesarean Section wound: A mini review. International Wound Journal, in press. 
CC (i) This work is licensed under Creative Commons Attribution 4.0 License

To Submit Your Article Click Here: $\quad$ Submit Article

DOI: $10.32474 /$ GJAPM.2020.03.000157

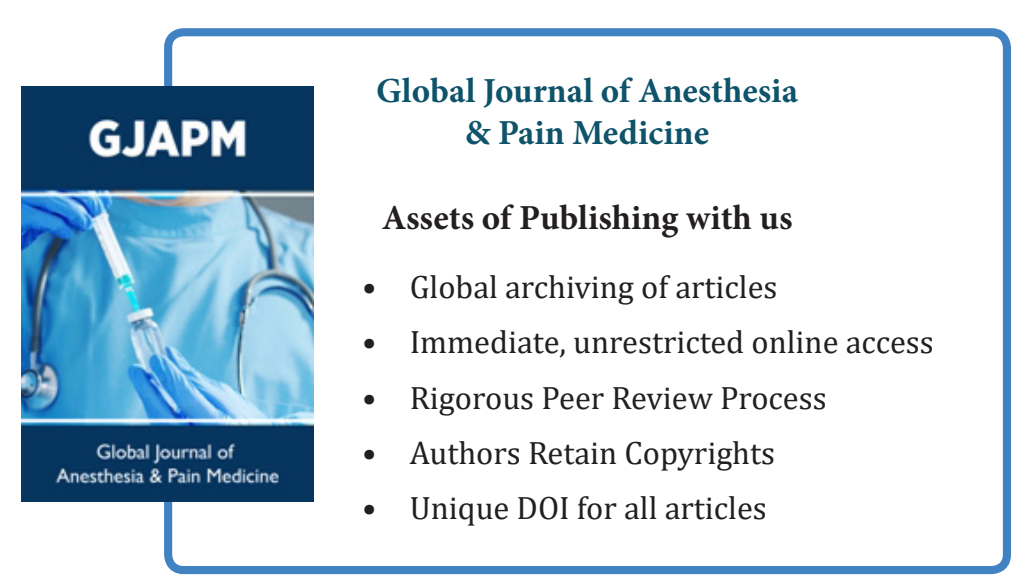

